\title{
Detecting chronic kidney disease in diabetic adults by estimating glomerular filtration rate and serum creatinine
}

\author{
Ahmed Kh. Mohammed Almaawi*
}

Senior Specialist Physician, Studies and Research Department, Commission of Medical Tertiary Centers, Ministry Of Health, Baghdad, Iraq.
*Correspondence to: Ahmed Kh. Mohammed Almaawi (E-mail: dr.ahmedaswad@gmail.com)
(Submitted: 28 November 2020 - Revised version received: 25 December 2020 - Accepted: 15 January 2021 - Published online: 26 February 2021)

\begin{abstract}
Objective To assess chronic kidney disease (CKD) prevalence and risk factors including socio-demography among diabetics by estimating glomerular filtration rate (GFR) rather than serum creatinine ( $\mathrm{sCr}$ ).

Methods A cross-sectional study was conducted in December 15, 2019 through August 15, 2020, among 800 diabetics attending tertiary diabetes centers, Baghdad. Data were collected by self-administered questionnaire. SPSS was used for data analysis by (mean, standard deviation and $t$-test) for quantitative variables and (frequency, percentage, Chi-square test and Kappa index) for qualitative variables. p-Value less than 0.05 was considered significant.

Results 800 diabetics for last 5-40 years, 95.6\% with type 2. Aged 52.1 \pm 13.2 years, with male:female ratio of 1.03:1, 63.6\% were with no income, sCr level was $0.86 \pm 0.3 \mathrm{mg} / \mathrm{dl}$, and eGFR by Cockcroft Gault (CG) and CKD-EPI equations was $100.4 \pm 36.5 \& 92.2 \pm 25.5$ $\mathrm{ml} / \mathrm{min} / 1.73 \mathrm{~m}^{2}$, respectively. CKD prevalence based on sCr, and eGFR assessed by above equations was $13.3 \%, 20 \%$ and $15.9 \%$, respectively $(p<0.001)$. Those with CKD were hypertensive, females, and living in peripheries.

Conclusions Diabetic patients, mainly those with risk factors are more likely to develop CKD. It is better to detect CKD initially by estimating the GFR, rather than sCr level alone. Furthermore, using CKD-EPI equation might be better than the CG formula to estimate the GFR.

Keywords Diabetes, Kidney, Creatinine
\end{abstract}

\section{Introduction}

Diabetes mellitus (DM) and chronic kidney disease (CKD) (as a part of non-communicable diseases) have replaced infection as a main cause of death in last century. DM is the seventh leading cause of death and responsible for $14.5 \%$ of global all-cause mortality. DM is an out of control pandemic, each year 10 million new cases are added globally. In Iraq, it affects 517,080 adults and expected to be $20,09,000$ by year 2030 , $46.5 \%$ of them are unaware of their status. ${ }^{1-9}$

DM may be subclinical for years; $25-50 \%$ of patients have complications at the time of diagnosis like diabetic nephropathy which is the leading cause of renal failure and increases death rate 20-40 times, rise in DM morbidity, mortality, and cost; is parallel to that of CKD., ${ }^{4,10-15}$

$\mathrm{CKD}$ is defined as drop in glomerular filtration rate (GFR) below $60 \mathrm{ml} / \mathrm{min} / 1.73 \mathrm{~m}^{2}$ for 3 months or more. Initial assessment should include GFR and not by serum creatinine $(\mathrm{sCr})$ alone. ${ }^{16-18}$. Staging based on GFR is a good way to assess CKD severity; stage 1 (GFR $\geq 90)$, stage 2 (60-89), stage 3 (30-59), stage $4(15-29)$, and stage $5(<15) .{ }^{17,19}$ Most of the diabetics with CKD will die from cardiovascular complication; death is two times more at stage 3 and three times at stage 4 than individuals with normal kidney function.,20,21 Many GFR estimating formulae are available like; Cockcroft Gault (CG), and CKD-epidemiology collaboration (CKD-EPI) (the best for diverse populations). ${ }^{22,23}$ In primary health care (PHC), most patients with CKD stages 1,2 are undetected as CKD is silent and reversible. GFR would be half normal before $\mathrm{sCr}$ level would be abnormal. Kidney Disease Improving Global Outcome (KDIGO) guidelines consider annual CKD initial screening by sCr-based estimated-GFR (eGFR) in diabetics as cost-effective; starting 5 years after diagnosis in type $1 \mathrm{DM}$, and at time of diagnosis in type 2 DM. Early detection, slow progression, decrease complications, and need for dialysis.
Primary care providers are well positioned to manage CKD patients as early referral to nephrologist improve prognosis but they are only detecting $10.8 \%$ of them at stage 3.,11,16,17,19,24-26 This study aims; to assess diabetic adults attending diabetes centers; socio-demographic profile, CKD prevalence, associated risk factors; and to increase family physicians awareness that GFR estimated by CKD-EPI equation is better than $\mathrm{sCr}$ alone to assess renal function and detect $\mathrm{CKD}$ among diabetic adults in PHC.

\section{Methods}

An outpatient-based cross-sectional study with some analytic elements conducted on registered diabetic adults attending diabetes and endocrine-related diseases tertiary centers in Baghdad (Rusafa \& Karkh) during the period from December 15, 2019 to August 15, 2020. Study project was reviewed and approved by Scientific and Ethics Committees of; Training \& Human Development National Center/ IMOH, and Baghdad/ Rusafa and Karkh Health Directorates, and informed consents were obtained from them. After satisfied study explanation, a verbal consent was obtained from all registered diabetic adults who attended study place during study period, met eligibility, and inclusion criteria, who consciously agreed to participate in study. Exclusion criteria were (age $<18$ or $>70$ years, diabetes for $<5$ years, body mass index (BMI) $>30$, (Cimetidine, Trimethoprim, Cephalosporin, Aspirin) medications in last 5 days, high meat meal last night, urinary tract structural anomaly, sickle cell anemia, spinal cord injury, or pregnancy). A convenience non-selective sampling method was used; researcher made regular study place visits for data collection in system of $5 \mathrm{~h}$ a day/ 1 day a week for 9 months. Based on eligibility criteria, 800 diabetic adults were recruited in study and data were collected from them. For each visit, 20-25 
patients were privately interviewed with their medical records checking. For data collection, a self-administered structured questionnaire-form paper was developed, and a pilot study had been done on a randomly selected 10 patients to figure out unclear questions and assess time needed to fill questionnaire. According to pilot study questionnaire, adjustment was done to be more acceptable, and patients who were subjected to pilot study weren't included in study. Questionnaire-form collect data; (age, gender, residence, occupation, income, marital status, smoking, diabetes type and duration, hypertension, heart disease, weight, height, BMI (weight $(\mathrm{kg}) / \mathrm{height}\left(\mathrm{m}^{2}\right)$ ), exclusion criteria, eGFR, and $\mathrm{sCr}$ ), sCr and eGFR values were used to detect CKD; $\mathrm{sCr} \geq 1.2 \mathrm{mg} / \mathrm{dl}$ for female, and $\geq 1.4 \mathrm{mg} /$ $\mathrm{dl}$ for male and eGFR (below $60 \mathrm{ml} / \mathrm{min} / 1.73 \mathrm{~m}^{2}$ estimated by standard CG and CKD-EPI equations) were considered as CKD. In order to compare results from both equations; values estimated by original CG equation $\left((140 \text {-age })^{\star}\right.$ weight/ $\mathrm{sCr}^{\star} 72\left({ }^{\star} 0.85\right.$ if female $\left.)\right)$ in $(\mathrm{ml} / \mathrm{min})$, were normalized to 1.73 $\mathrm{m}^{2}$ body surface area by multiplying GFR by $\left(1.73 /\right.$ (weight $^{0.4}$ ${ }^{25 *}$ height $\left.{ }^{0.725}\right)^{\star} 0.007184$ ) according to Du Bois equation. ${ }^{22,23,27}$ For data entry, storage, and analysis, computerized software SPSS 20 IBM (Statistical Package for Social Science version 20) was used. Data were stratified according to age, eGFR, and CKD. Mean, standard deviation, and $t$-test were used to present continuous variables and to check association significance. Frequency and percentage, Chi-square test, and kappa index cross-tabulation were used to present discrete variables and to check association significance. Probability (p-values) of less 0.05 was considered as statistically significant.

\section{Results}

Study included 800 adults with DM for the last $11.7 \pm 6.9(5-40)$ years (yr), majority $95.6 \%$ (765) with type $2 \mathrm{DM}$, and only $4.4 \%$ (35) with type 1, aged 52.1 $\pm 13.2(18-70)$ yr; (53.75\%(430) aged 41-60 yr, 28.38\%(227) aged 61-70 yr and 17.87\% (143) aged 18-40 yr). Study included 405 (50.6\%) males and 395 (49.4\%) females, with male:female ratio was $1.03: 1.88 .4 \%$ (707) of patients live in nearby places. Table 1 shows that $90.5 \%$ (724) of patients are married while 9.5\% (76) were either single, divorced, or widow. 63.6\%(509) with no income, and only $31.9 \%(255)$ had fixed monthly income salary (from a job $16.5 \%(132)$, retirement $13.3 \%(106)$, or governmental allowance $2.1 \%(17)$ ), and $4.5 \%(36)$ had no fixed income. $90.9 \%$ (727) had secondary school educational level or less and only $9.1 \%$ (73) had a college level or above. $14.8 \%$ (128) of them were smokers. The mean BMI of study group was $(25.6 \pm 2.6$ (16.730) $\mathrm{kg} / \mathrm{m}^{2}$ ). Table 2 shows that mean sCr level was $0.86 \pm 0.3$ $\mathrm{mg} / \mathrm{dl}$, mean eGFR detected by CG and CKD-EPI equations was $100.4 \pm 36.5 \& 92.2 \pm 25.5 \mathrm{ml} / \mathrm{min} / 1.73 \mathrm{~m}^{2}$, respectively $(\mathrm{p}<0.001)$. CKD prevalence detected by abnormal $\mathrm{sCr}$ was $13.3 \%(106 / 800)(57 / 800$ (7.13\%) females and 49/800 (6.13\%) males, $\mathrm{p}=0.045)$. This is significantly different from CKD prevalence by using CG equation $20 \%(160 / 800)(p<0.001)$, and using CKD-EPI equation 15.9\%(127/800), results by CG was higher than that by CKD-EPI equation $(\mathrm{p}<0.001)$. According to CKD-EPI, $61.4 \%(78 / 127)$ of those with CKD aged $61-70$ yr. CKD prevalence among males aged between 61 and $70 \mathrm{yr}$ was higher by using CG than CKD-EPI equation $(\mathrm{p}<0.001)$.

\begin{tabular}{|c|c|c|c|c|c|c|c|c|c|c|}
\hline \multirow{3}{*}{$\begin{array}{l}\text { Age } \\
\text { (yr) }\end{array}$} & \multirow{3}{*}{ Gender } & \multirow{3}{*}{$\begin{array}{c}\text { Married } \\
\text { n. } \%\end{array}$} & \multicolumn{5}{|c|}{ Income n. \% } & \multirow{2}{*}{\multicolumn{2}{|c|}{$\begin{array}{c}\text { Education } \\
\text { n. } \%\end{array}$}} & \multirow{3}{*}{$\begin{array}{c}\text { BMI } \\
\mathbf{k g} / \mathrm{m}^{2} \\
\text { Mean } \pm \text { SD } \\
\text { (Range) }\end{array}$} \\
\hline & & & \multicolumn{3}{|c|}{ Fixed income } & \multirow{2}{*}{ 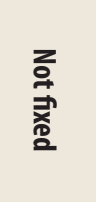 } & \multirow{2}{*}{$\begin{array}{l}z \\
\text { zo. } \\
\overline{\overline{0}} \\
\overline{0} \\
\overline{0}\end{array}$} & & & \\
\hline & & & 흥 & 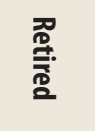 & ฏ & & & 글 & $\frac{\varrho}{\overline{0}}$ & \\
\hline \multirow{2}{*}{$18-40$} & $\begin{array}{l}\text { Male } \\
\text { No. }=66\end{array}$ & $\begin{array}{c}39 \\
59.1 \%\end{array}$ & $\begin{array}{c}23 \\
34.8 \%\end{array}$ & $\begin{array}{c}0 \\
0 \%\end{array}$ & $\begin{array}{c}1 \\
1.5 \%\end{array}$ & $\begin{array}{c}11 \\
16.7 \%\end{array}$ & $\begin{array}{c}31 \\
47 \%\end{array}$ & $\begin{array}{c}54 \\
81.8 \%\end{array}$ & $\begin{array}{c}12 \\
18.2 \%\end{array}$ & $\begin{array}{c}24.4 \pm 2.8 \\
(17.3-29.8)\end{array}$ \\
\hline & $\begin{array}{l}\text { Female } \\
\text { No. }=77\end{array}$ & $\begin{array}{c}50 \\
64.9 \%\end{array}$ & $\begin{array}{c}7 \\
9.1 \%\end{array}$ & $\begin{array}{c}0 \\
0 \%\end{array}$ & $\begin{array}{c}1 \\
1.3 \%\end{array}$ & $\begin{array}{c}2 \\
2.6 \%\end{array}$ & $\begin{array}{c}67 \\
87 \%\end{array}$ & $\begin{array}{c}65 \\
84.4 \%\end{array}$ & $\begin{array}{c}12 \\
15.6 \%\end{array}$ & $\begin{array}{l}25.2 \pm 2.3 \\
(19.5-30)\end{array}$ \\
\hline \multirow{2}{*}{$41-60$} & $\begin{array}{l}\text { Male } \\
\text { No=209 }\end{array}$ & $\begin{array}{c}208 \\
99.5 \%\end{array}$ & $\begin{array}{c}65 \\
31.1 \%\end{array}$ & $\begin{array}{c}28 \\
13.4 \%\end{array}$ & $\begin{array}{c}8 \\
3.8 \%\end{array}$ & $\begin{array}{c}18 \\
8.6 \%\end{array}$ & $\begin{array}{c}90 \\
43.1 \%\end{array}$ & $\begin{array}{l}188 \\
90 \%\end{array}$ & $\begin{array}{c}21 \\
10 \%\end{array}$ & $\begin{array}{c}25.4 \pm 2.6 \\
(19.2-29.98)\end{array}$ \\
\hline & $\begin{array}{l}\text { Female } \\
\text { No }=221\end{array}$ & $\begin{array}{c}209 \\
94.6 \%\end{array}$ & $\begin{array}{c}18 \\
8.1 \%\end{array}$ & $\begin{array}{c}6 \\
2.7 \%\end{array}$ & $\begin{array}{c}0 \\
0 \%\end{array}$ & $\begin{array}{c}1 \\
0.5 \%\end{array}$ & $\begin{array}{c}196 \\
88.7 \%\end{array}$ & $\begin{array}{c}209 \\
94.6 \%\end{array}$ & $\begin{array}{c}12 \\
5.4 \%\end{array}$ & $\begin{array}{l}26.1 \pm 2.6 \\
(18.3-30)\end{array}$ \\
\hline \multirow{2}{*}{$61-70$} & $\begin{array}{l}\text { Male } \\
\text { No=130 }\end{array}$ & $\begin{array}{c}127 \\
97.7 \%\end{array}$ & $\begin{array}{c}16 \\
12.3 \%\end{array}$ & $\begin{array}{c}65 \\
50 \%\end{array}$ & $\begin{array}{c}4 \\
3.1 \%\end{array}$ & $\begin{array}{c}4 \\
3.1 \%\end{array}$ & $\begin{array}{c}41 \\
31.5 \%\end{array}$ & $\begin{array}{c}115 \\
88.5 \%\end{array}$ & $\begin{array}{c}15 \\
11.5 \%\end{array}$ & $\begin{array}{l}25.2 \pm 2.5 \\
(16.7-30)\end{array}$ \\
\hline & $\begin{array}{l}\text { Female } \\
\text { No. }=97\end{array}$ & $\begin{array}{c}91 \\
93.8 \%\end{array}$ & $\begin{array}{c}3 \\
3.1 \%\end{array}$ & $\begin{array}{c}7 \\
7.2 \%\end{array}$ & $\begin{array}{c}3 \\
3.1 \%\end{array}$ & $\begin{array}{c}0 \\
0 \%\end{array}$ & $\begin{array}{c}84 \\
86.6 \%\end{array}$ & $\begin{array}{c}96 \\
99 \%\end{array}$ & $\begin{array}{c}1 \\
1 \%\end{array}$ & $\begin{array}{l}26.3 \pm 2.8 \\
(19.5-30)\end{array}$ \\
\hline \multirow{3}{*}{$18-70$} & $\begin{array}{l}\text { Male } \\
\text { No }=405\end{array}$ & $\begin{array}{c}374 \\
92.3 \%\end{array}$ & $\begin{array}{c}104 \\
25.7 \%\end{array}$ & $\begin{array}{c}93 \\
23 \%\end{array}$ & $\begin{array}{c}13 \\
3.2 \%\end{array}$ & $\begin{array}{c}33 \\
8.1 \%\end{array}$ & $\begin{array}{l}162 \\
40 \%\end{array}$ & $\begin{array}{c}357 \\
88.1 \%\end{array}$ & $\begin{array}{c}48 \\
11.9 \%\end{array}$ & $\begin{array}{c}25.2 \pm 2.6 \\
(16.7-29.98)\end{array}$ \\
\hline & $\begin{array}{l}\text { Female } \\
\text { No }=395\end{array}$ & $\begin{array}{c}350 \\
88.6 \%\end{array}$ & $\begin{array}{c}28 \\
7.1 \%\end{array}$ & $\begin{array}{c}13 \\
3.3 \%\end{array}$ & $\begin{array}{c}4 \\
1 \%\end{array}$ & $\begin{array}{c}3 \\
0.8 \%\end{array}$ & $\begin{array}{c}347 \\
87.8 \%\end{array}$ & $\begin{array}{c}370 \\
93.7 \%\end{array}$ & $\begin{array}{l}25 \\
6.3 \%\end{array}$ & $\begin{array}{c}26 \pm 2.6 \\
(18.3-30)\end{array}$ \\
\hline & $\begin{array}{l}\text { Total } \\
\mathrm{N}=800\end{array}$ & $\begin{array}{c}724 \\
90.5 \%\end{array}$ & $\begin{array}{c}132 \\
16.5 \%\end{array}$ & $\begin{array}{c}106 \\
13.3 \%\end{array}$ & $\begin{array}{c}17 \\
2.1 \%\end{array}$ & $\begin{array}{c}36 \\
4.5 \%\end{array}$ & $\begin{array}{c}509 \\
63.6 \%\end{array}$ & $\begin{array}{c}727 \\
90.9 \%\end{array}$ & $\begin{array}{c}73 \\
9.1 \%\end{array}$ & $\begin{array}{l}25.6 \pm 2.6 \\
(16.7-30)\end{array}$ \\
\hline
\end{tabular}

n.: number of subjects with specific characteristic; \%: Percentage $=(\mathrm{n} . /$ No); yr: year; SD: standard deviation; BMI: body mass index 
Table 2. Serum creatinine, eGFR and CKD prevalence of study sample in different age groups ( $\mathrm{N}$ : total sample size $=\mathbf{8 0 0}$ ).

\begin{tabular}{|c|c|c|c|c|c|c|c|c|}
\hline \multirow[b]{2}{*}{$\begin{array}{l}\text { Age } \\
\text { (yr) }\end{array}$} & \multirow[b]{2}{*}{ Gender } & \multicolumn{2}{|c|}{ Serum Creatinine } & \multicolumn{2}{|c|}{ CKD-EPI equation } & \multicolumn{2}{|c|}{ CG equation } & \multirow[b]{2}{*}{$P$ value } \\
\hline & & $\begin{array}{c}\text { s.Cr } \\
\text { Mean } \pm S D \\
\text { Range }\end{array}$ & $\begin{array}{c}\text { CKD } \\
\text { Prevalence } \\
\text { n. } \%\end{array}$ & $\begin{array}{c}\text { eGFR } \\
\text { Mean } \pm S D \\
\text { Range }\end{array}$ & $\begin{array}{c}\text { CKD } \\
\text { Prevalence } \\
\text { n. } \%\end{array}$ & $\begin{array}{c}\text { eGFR } \\
\text { Mean } \pm \text { SD } \\
\text { Range }\end{array}$ & $\begin{array}{c}\text { CKD } \\
\text { Prevalence } \\
\text { n. } \%\end{array}$ & \\
\hline \multirow{2}{*}{$18-40$} & $\begin{array}{l}\text { Male } \\
\text { No. }=66\end{array}$ & $\begin{array}{c}0.81 \pm 0.28 \\
0.5-2.15\end{array}$ & $\begin{array}{c}5 \\
7.6 \%\end{array}$ & $\begin{array}{c}119.2 \pm 24.3 \\
41.6-161\end{array}$ & $\begin{array}{c}1 \\
1.5 \%\end{array}$ & $\begin{array}{c}137.4 \pm 37.6 \\
54-237.8\end{array}$ & $\begin{array}{c}1 \\
1.5 \%\end{array}$ & 0.076 \\
\hline & $\begin{array}{l}\text { Female } \\
\text { No. }=77\end{array}$ & $\begin{array}{c}0.71 \pm 0.26 \\
0.3-1.42\end{array}$ & $\begin{array}{c}10 \\
13 \%\end{array}$ & $\begin{array}{c}111.3 \pm 27 \\
47.6-165.4\end{array}$ & $\begin{array}{c}5 \\
6.5 \%\end{array}$ & $\begin{array}{c}137.1 \pm 45.9 \\
59.7-323.1\end{array}$ & $\begin{array}{c}2 \\
2.6 \%\end{array}$ & 0.015 \\
\hline \multirow{2}{*}{$41-60$} & $\begin{array}{l}\text { Male } \\
\text { No=209 }\end{array}$ & $\begin{array}{l}0.9 \pm 0.3 \\
0.5-3.9\end{array}$ & $\begin{array}{c}15 \\
7.2 \%\end{array}$ & $\begin{array}{l}96.4 \pm 18.3 \\
15.9-128.2\end{array}$ & $\begin{array}{c}14 \\
6.7 \%\end{array}$ & $\begin{array}{c}100.9 \pm 25 \\
21.04-167.4\end{array}$ & $\begin{array}{c}19 \\
9.1 \%\end{array}$ & $<0.001$ \\
\hline & $\begin{array}{l}\text { Female } \\
\text { No }=221\end{array}$ & $\begin{array}{c}0.74 \pm 0.23 \\
0.34-1.6\end{array}$ & $\begin{array}{c}23 \\
10.4 \%\end{array}$ & $\begin{array}{c}92.6 \pm 20 \\
37.3-124.1\end{array}$ & $\begin{array}{c}29 \\
13.1 \%\end{array}$ & $\begin{array}{l}104.6 \pm 29.3 \\
40.8-189.7\end{array}$ & $\begin{array}{c}29 \\
13.1 \%\end{array}$ & $<0.001$ \\
\hline \multirow{2}{*}{$61-70$} & $\begin{array}{l}\text { Male } \\
\text { No=130 }\end{array}$ & $\begin{array}{c}1.07 \pm 0.43 \\
0.54-4 .-8\end{array}$ & $\begin{array}{c}29 \\
22.3 \%\end{array}$ & $\begin{array}{l}76.8 \pm 21.5 \\
14.1-111.9\end{array}$ & $\begin{array}{c}38 \\
29.2 \%\end{array}$ & $\begin{array}{l}73.9 \pm 22.5 \\
14.8-134.6\end{array}$ & $\begin{array}{c}57 \\
43.8 \%\end{array}$ & $<0.001$ \\
\hline & $\begin{array}{l}\text { Female } \\
\text { No. }=97\end{array}$ & $\begin{array}{c}0.94 \pm 0.34 \\
0.47-2.33\end{array}$ & $\begin{array}{c}24 \\
24.7 \%\end{array}$ & $\begin{array}{l}69.1 \pm 21.9 \\
20.6-105.5\end{array}$ & $\begin{array}{c}40 \\
41.2 \%\end{array}$ & $\begin{array}{l}71.2 \pm 23.9 \\
26.2-129.6\end{array}$ & $\begin{array}{c}52 \\
53.6 \%\end{array}$ & $<0.001$ \\
\hline \multirow{3}{*}{$18-70$} & $\begin{array}{l}\text { Male } \\
\text { No=405 }\end{array}$ & $\begin{array}{c}0.94 \pm 0.36 \\
0.5-4.08\end{array}$ & $\begin{array}{c}49 \\
12.1 \%\end{array}$ & $\begin{array}{c}93.8 \pm 24.8 \\
14.1-161\end{array}$ & $\begin{array}{c}53 \\
13.1 \%\end{array}$ & $\begin{array}{c}98.2 \pm 34 \\
14.8-237.8\end{array}$ & $\begin{array}{c}77 \\
19 \%\end{array}$ & $<0.001$ \\
\hline & $\begin{array}{l}\text { Female } \\
\mathrm{No}=395\end{array}$ & $\begin{array}{c}0.78 \pm 0.28 \\
0.3-2.33\end{array}$ & $\begin{array}{c}57 \\
14.4 \%\end{array}$ & $\begin{array}{l}90.5 \pm 26.1 \\
20.6-165.4\end{array}$ & $\begin{array}{c}74 \\
18.7 \%\end{array}$ & $\begin{array}{c}102.8 \pm 38.8 \\
26.2-323.1\end{array}$ & $\begin{array}{c}83 \\
21 \%\end{array}$ & $<0.001$ \\
\hline & $\begin{array}{l}\text { Total } \\
\mathrm{N}=800\end{array}$ & $\begin{array}{c}0.86 \pm 0.3 \\
0.3-4.08\end{array}$ & $\begin{array}{c}106 \\
13.3 \%\end{array}$ & $\begin{array}{l}92.2 \pm 25.5 \\
14.1-165.4\end{array}$ & $\begin{array}{c}127 \\
15.9 \%\end{array}$ & $\begin{array}{c}100.4 \pm 36.5 \\
14.8-323.1\end{array}$ & $\begin{array}{l}160 \\
20 \%\end{array}$ & $<0.001$ \\
\hline
\end{tabular}

n.: number of subjects with specific characteristic; \%: Percent = (n. /No); SD: Standard Deviation; s.Cr: Serum Creatinine; yr: Year; eGFR: estimated Glomerular

Filtration Rate in (ml/min/1.73 m²) units; CKD-EPI: Chronic Kidney Disease Epidemiology equation; CG: Cockcroft Gault equation; Abnormal s.Cr: $\geq 1.4 m g / d l$ in male: $\geq 1.2 \mathrm{mg} / \mathrm{dl}$ in female; Abnormal eGFR: less than $60 \mathrm{ml} / \mathrm{min} / 1.73 \mathrm{~m}^{2}$

To study different associated risk factors, sample was divided into two groups; with or without CKD (defined by eGFR level of less than $60 \mathrm{ml} / \mathrm{min} / 1.73 \mathrm{~m}^{2}$ detected by CKD-EPI). As shown in Table 3, 265/800 (33.1\%) patients were hypertensive for the last $1-32$ years, and $48 / 800(6 \%)$ gave a history of heart disease for the last 1-25 years. Those with CKD were more likely to be hypertensive $(\mathrm{p}<0.001)$, female $(\mathrm{p}=0.029)$, and lived in periphery $(\mathrm{p}<0.001)$, and more likely to be older, less educated with heart disease and no income. To compare CG and CKD-EPI equations, sample was classified into five stages by eGFR level estimated by using two equations as shown in Table 4; comparing result from each stage showed significant difference ( $\mathrm{p}<0.001)$. According to CKD-EPI, 122/127 (96.1\%) of patients with CKD were in stage 3. CKD (stage 3 ) prevalence was higher by using CG while stage 4 prevalence was higher by CKD-EPI. Table 5 shows that cross-tabulating results of both equations showed a strong agreement; $684(85.5 \%)$ and by using kappa index (kappa value: $0.73,95 \%$ confidence interval (CI): 0.68-0.77, $\mathrm{p}<0.001)$.

CKD-EPI: Chronic Kidney Disease Epidemiology equation; CG: Cockcroft Gault equation; eGFR: estimated Glomerular Filtration Rate; n: Number of subjects with specific character; \%: Percent $=(\mathrm{n} / \mathrm{N})$.

\section{Discussion}

This study aims to assess CKD prevalence in diabetic adults and to check whether estimating eGFR using CKD-EPI formula can detect CKD better than CG formula or sCr level.
CKD screening is cost-effective as earlier intervention can slow renal damage progression and serves oriented family physician to use modest PHC resources judgmentally where $\mathrm{sCr}$ is feasible, albuminuria is not; but CKD is underestimated whether by PHC providers or by International Classification of Diseases-9(ICD-9). ${ }^{4,24,28,29}$ To achieve this aim, 800 patients' data were analyzed regarding socio-demography, risk factors, and CKD prevalence in different age groups and stages. The study diabetic population was with 1.03:1 male:female ratio and was middle aged, overweighed, modestly educated, with no job or fixed income, and lived in nearby city areas. This in agreement with Narenpitak et al where $760 \mathrm{DM}$ patients aged $58.7 \pm 9.8$ yr. with BMI $25.6 \pm 4.2 \mathrm{~kg} / \mathrm{m}^{2} .{ }^{30}$ Both genders are somewhat equally affected by DM, but obese people are 80 times more likely to be affected. In Iraq, genetics, socio-demographic changes, urbanization, increasing sedentary lifestyle, and overweight had led to emerging DM epidemics mainly in age above $40 ., 4,8,10,12$ CKD prevalence in this study was significantly higher by using CG equation (20\%) than with CKD-EPI (15.9\%), or abnormal sCr level (13.3\%). This is in agreement with Zaman et al; CKD prevalence by using CG (31.4\%), by CKD-EPI (21.9\%) and by sCr (18.6\%) though prevalence was higher as the last is a hospital-based and ours is outpatient-based study. ${ }^{31}$ And in agreement with Bouzid et al; prevalence using CG: $19.8 \%^{32}$ and Fiseha et al; prevalence using CG: $23.8 \% .^{33}$ All this resembles a worldwide over/underestimation of CKD with a wide variation depending on the used approach, obesity, high age, economic problems, and DM prevalence. ${ }^{15,19,34}$ In $\mathrm{PHC}$ sCr isn't enough for renal function 


\begin{tabular}{|c|c|c|c|c|c|c|c|}
\hline \multirow{3}{*}{$\begin{array}{l}\text { Associated Risk } \\
\text { Factor }\end{array}$} & \multicolumn{6}{|c|}{ Presence of CKD } & \multirow{3}{*}{$\begin{array}{c}\text { p. } \\
\text { value }\end{array}$} \\
\hline & \multicolumn{3}{|c|}{ With CKD } & \multicolumn{3}{|c|}{ Without CKD } & \\
\hline & $\begin{array}{c}\text { Male } \\
\text { No. }=53\end{array}$ & $\begin{array}{l}\text { Female } \\
\text { No.=74 }\end{array}$ & $\begin{array}{c}\text { Total } \\
\text { No.=127 }\end{array}$ & $\begin{array}{c}\text { Male } \\
\text { No.=352 }\end{array}$ & $\begin{array}{l}\text { Female } \\
\text { No.=321 }\end{array}$ & $\begin{array}{c}\text { Total } \\
\text { No. }=673\end{array}$ & \\
\hline $\begin{array}{l}\text { Hypertension } \\
\text { n. \% }\end{array}$ & $\begin{array}{c}29 \\
45.7 \%\end{array}$ & $\begin{array}{c}39 \\
52.7 \%\end{array}$ & $\begin{array}{c}68 \\
53.5 \%\end{array}$ & $\begin{array}{c}87 \\
24.7 \%\end{array}$ & $\begin{array}{c}110 \\
34.3 \%\end{array}$ & $\begin{array}{c}197 \\
29.3 \%\end{array}$ & $<0.001$ \\
\hline $\begin{array}{l}\text { Heart disease } \\
\text { n. \% }\end{array}$ & $\begin{array}{c}4 \\
7.5 \%\end{array}$ & $\begin{array}{c}6 \\
8.1 \%\end{array}$ & $\begin{array}{c}10 \\
7.9 \%\end{array}$ & $\begin{array}{c}19 \\
5.4 \%\end{array}$ & $\begin{array}{c}19 \\
5.9 \%\end{array}$ & $\begin{array}{c}38 \\
5.6 \%\end{array}$ & 0.332 \\
\hline $\begin{array}{l}\text { Female } \\
\text { n. } \%\end{array}$ & - & $\begin{array}{c}74 \\
100 \%\end{array}$ & $\begin{array}{c}74 \\
58.3 \%\end{array}$ & - & $\begin{array}{c}321 \\
100 \%\end{array}$ & $\begin{array}{c}673 \\
47.7 \%\end{array}$ & 0.029 \\
\hline $\begin{array}{l}\text { Periphery living } \\
\text { n. \% }\end{array}$ & $\begin{array}{c}4 \\
7.5 \%\end{array}$ & $\begin{array}{c}24 \\
32.4 \%\end{array}$ & $\begin{array}{c}28 \\
22 \%\end{array}$ & $\begin{array}{l}28 \\
8 \%\end{array}$ & $\begin{array}{c}37 \\
11.5 \%\end{array}$ & $\begin{array}{c}65 \\
9.7 \%\end{array}$ & $<0.001$ \\
\hline $\begin{array}{l}\text { 2ndary School or less } \\
\text { n. \% }\end{array}$ & $\begin{array}{c}40 \\
75.5 \%\end{array}$ & $\begin{array}{c}71 \\
95.9 \%\end{array}$ & $\begin{array}{c}111 \\
87.4 \%\end{array}$ & $\begin{array}{c}317 \\
90.1 \%\end{array}$ & $\begin{array}{c}299 \\
93.1 \%\end{array}$ & $\begin{array}{c}616 \\
91.5 \%\end{array}$ & 0.138 \\
\hline $\begin{array}{l}\text { Smoking } \\
\text { n. \% }\end{array}$ & $\begin{array}{c}15 \\
28.3 \%\end{array}$ & $\begin{array}{l}3 \\
4.1 \%\end{array}$ & $\begin{array}{c}18 \\
14.2 \%\end{array}$ & $\begin{array}{c}98 \\
27.8 \%\end{array}$ & $\begin{array}{c}2 \\
0.6 \%\end{array}$ & $\begin{array}{c}100 \\
14.9 \%\end{array}$ & 0.842 \\
\hline $\begin{array}{l}\text { No income } \\
\text { n. } \%\end{array}$ & $\begin{array}{c}19 \\
35.8 \%\end{array}$ & $\begin{array}{c}63 \\
85.1 \%\end{array}$ & $\begin{array}{c}82 \\
64.6 \%\end{array}$ & $\begin{array}{c}143 \\
40.6 \%\end{array}$ & $\begin{array}{c}284 \\
88.5 \%\end{array}$ & $\begin{array}{c}472 \\
63.4 \%\end{array}$ & 0.81 \\
\hline
\end{tabular}

n.: number of subjects with specific characteristic; \%: Percentage= (n. /No); DM: Diabetes Mellitus, yr: Year; SD: Standard Deviation; CKD: Chronic Kidney Disease; eGFR less than $60 \mathrm{ml} / \mathrm{min} / 1.73 \mathrm{~m}^{2}$ detected by CKD-EPI equation.

\begin{tabular}{|c|c|c|c|c|c|}
\hline CKD stage & $\begin{array}{c}\text { eGFR } \\
\left(\mathrm{ml} / \mathrm{min} / 1.73 \mathrm{~m}^{2}\right)\end{array}$ & Gender & $\begin{array}{l}\text { CKD Prevalence by } \\
\text { CKD-EPI equation } \\
\qquad N=800\end{array}$ & $\begin{array}{l}\text { CKD Prevalence } \\
\text { by CG equation } \\
\qquad \mathrm{N}=800\end{array}$ & p. value \\
\hline \multirow{2}{*}{1} & \multirow{2}{*}{$>90$} & Male n. (\%) & $284(35.5 \%)$ & 234 (29.3\%) & \multirow{2}{*}{$<0.001$} \\
\hline & & Female n. (\%) & $252(31.5 \%)$ & $246(30.8 \%)$ & \\
\hline \multirow{2}{*}{2} & \multirow{2}{*}{$60-89$} & Male n. (\%) & $68(8.5 \%)$ & $94(11.8 \%)$ & \multirow{2}{*}{$<0.001$} \\
\hline & & Female n. (\%) & $69(8.6 \%)$ & $66(8.3 \%)$ & \\
\hline \multirow{2}{*}{3} & \multirow{2}{*}{$30-59$} & Male n. (\%) & $51(6.4 \%)$ & 75 (9.4\%) & \multirow{2}{*}{$<0.001$} \\
\hline & & Female n. (\%) & $71(8.9 \%)$ & 81 (10.1\%) & \\
\hline \multirow{2}{*}{4} & \multirow{2}{*}{$15-29$} & Male n. (\%) & $1(0.1 \%)$ & $1(0.1 \%)$ & \multirow{2}{*}{$<0.001$} \\
\hline & & Female n. (\%) & $3(0.4 \%)$ & $2(0.3 \%)$ & \\
\hline \multirow{2}{*}{5} & \multirow{2}{*}{$<15$} & Male n. (\%) & $1(0.1 \%)$ & $1(0.1 \%)$ & \multirow{2}{*}{-} \\
\hline & & Female n. (\%) & $0(0 \%)$ & $0(0 \%)$ & \\
\hline \multirow{2}{*}{$3-5$} & \multirow{2}{*}{$<60$} & Male n. (\%) & $53(6.6 \%)$ & 77 (9.6\%) & \multirow{2}{*}{0.001} \\
\hline & & Female n. (\%) & 74 (9.3\%) & $83(10.4 \%)$ & \\
\hline
\end{tabular}

CKD-EPI: Chronic Kidney Disease Epidemiology equation; CG: Cockcroft Gault equation; eGFR: estimated Glomerular Filtration Rate; n.: number of subjects with characteristic; \%: Percent= (n. /N).

assessment as its level will keep normal till renal function drop by $50 \%,{ }^{19,25}$ compared to measured GFR; estimating eGFR have systematic bias but it is minimal (10-20\%) in CG and CKD-EPI. CG formula is affected by weight while CKD-EPI is the most accurate one for diverse population. ${ }^{18,23}$ Majority (61.4\%) of CKD (by CKD-EPI) were in patients aged 61-70 yr which is in agreement with Nakata. ${ }^{35}$ This is expected as there is continuous renal function drop after age of $30 \mathrm{yr}$, reaching a CKD prevalence of $25 \%$ by age of $70 .{ }^{16,17}$ In order to study CKD provoking factors, sample was divided into two groups; with and without $\mathrm{CKD}$, hypertension prevalence was higher in those with CKD, in agreement with Bradshaw et al, Narenpitak et al, Hooi et al, and Jolly et al; DM and hypertension are independent risk factors and responsible for $15 \%$ of CKD.,30,36-39 


\begin{tabular}{|c|c|c|c|c|c|c|c|}
\hline & & \multicolumn{5}{|c|}{ CKD staging by CKD-EPI n (\%) } & \multirow{2}{*}{ Total } \\
\hline & & 1 & 2 & 3 & 4 & 5 & \\
\hline \multirow{5}{*}{$\begin{array}{l}\text { CKD staging } \\
\text { by CG } \\
\text { n (\%) }\end{array}$} & 1 & 472 (59\%) & $8(1 \%)$ & $0(0 \%)$ & $0(0 \%)$ & $0(0 \%)$ & $480(60 \%)$ \\
\hline & 2 & $64(8 \%)$ & 91 (11.4\%) & $5(0.6 \%)$ & $0(0 \%)$ & $0(0 \%)$ & $160(20 \%)$ \\
\hline & 3 & $0(0 \%)$ & $38(4.8 \%)$ & $117(14.6 \%)$ & $1(0.1 \%)$ & $0(0 \%)$ & $156(19.5 \%)$ \\
\hline & 4 & $0(0 \%)$ & $0(0 \%)$ & $0(0 \%)$ & $3(0.4 \%)$ & $0(0 \%)$ & $3(0.4 \%)$ \\
\hline & 5 & $0(0 \%)$ & $0(0 \%)$ & $0(0 \%)$ & $0(0 \%)$ & $1(0.1 \%)$ & $1(0.1 \%)$ \\
\hline Total & & 536 (67\%) & 137 (17.1\%) & $122(15.2 \%)$ & $4(0.5 \%)$ & $1(0.1 \%)$ & $800(100 \%)$ \\
\hline
\end{tabular}

CKD-EPI: Chronic Kidney Disease Epidemiology equation; CG: Cockcroft Gault equation; eGFR: estimated Glomerular Filtration Rate; n: Number of subjects with specific character; \%: Percent $=(n / N)$.

Heart disease prevalence was higher in those with CKD in agreement with Bradshaw et al and (Jolly et al; cardiovascular risk increase as eGFR drop, death related to heart disease was 2-3 times in those with stage 3 and $4 \mathrm{CKD}$, respectively, than that with normal kidney function. ${ }^{4,21,36,38}$ Those with CKD are more likely to be unemployed with no income which is in agreement with Bradshaw et al. Females' percent was higher in those with CKD which is in agreement with Hooi et $\mathrm{al}^{37}$; as age-related diabetic kidney disease progression differ between sexes. ${ }^{40}$ Those living in periphery are more likely to have CKD; in low income countries where unaffordable costly health service, unawareness, ${ }^{41}$ unemployment and DM epidemic in Iraq, making CKD a leading cause of death and lately referred CKD. ${ }^{36,42-44}$ Study sample was categorized to five stages according to eGFR level ${ }^{19}$ using two equations; standard CKD-EPI (the most accepted index one $\mathrm{e}^{23}$ ) and CG, comparing between above equations showed strong agreement: $85.5 \%$, kappa: 0.73 (95\% CI: $0.68-0.77, \mathrm{p}<0.001$ ), $96.1 \%$ of CKD patients were with stage 3 that was higher by depending on CG which is in agreement with Zaman et al that found strong agreement: 70.9\%, kappa: 0.56 (95\% CI: 0.44-0.67, p < 0.001) and considered high CG prevalence as overestimation compared to CKD-EPI ${ }^{31}$ and in agreement with Kitiyakara et al $2012 ; ;^{45}$ that is related to continuously increasing CKD (stage 3) prevalence among adult in correlation with increasing DM, advanced age, and obesity. ${ }^{19}$ CG doesn't take diverse people in consideration and requires weight and height which isn't available in laboratories while CKD-EPI does not; CKD-EPI is better to assess CKD prevalence in diabetics despite strong agreement between them.

\section{Conclusion}

Diabetic adults especially those with risk factors are likely to develop CKD and it is better to detect CKD initially in PHC and in tertiary centers by estimating GFR rather than depending on $\mathrm{SCr}$ alone. CKD-EPI formula may be better than CG to achieve that goal.

\section{Recommendations}

- Family physicians, general practitioners, and PHC providers should be aware for CKD among risky patients including diabetics attending PHC centers.

- Initial CKD screening for risky patients should be done by eGFR estimation using a creatinine-based formula rather than $\mathrm{sCr}$ alone.

- Family physicians are in the best position for CKD detection, initial management, and follow up in coordination with nephrologist.

\section{References}

1. Omran A. The epidemiologic transition: a theory of the epidemiology of population change. Milbank Q 2005:83(4);731-757. Available at: https:// www.ncbi.nlm.nih.gov/pmc/articles/PMC2690264/\#

2. Stenvinkel P. Chronic kidney disease: a public health priority and harbinge of premature cardiovascular disease. J Int Med 2010;268:456-467. Available at: https://onlinelibrary.wiley.com/doi/full/10.1111/j.1365-2796.2010.02269.

3. Alwan A. Chronic non-communicable diseases. In: Health in Iraq: The Current Situation, Our Vision for the Future and Areas of Work, $2^{\text {nd }}$ ed. BGD (Iraq): Al-Adib Press; 2004;31-40. Available online at: https://www.who.int/ hac/crises/irq/background/Iraq_Health_in_Iraq_second_edition.pdf?ua=1.

4. Bakris G, Ritz E. The message for World Kidney Day 2009: hypertension and kidney disease - a marriage that should be prevented. J Hum Hypertens 2009;23:222-225. Available at: https://www.nature.com/articles/ jhh2008169.

5. Pearson ER, McCrimmon RJ. Diabetes mellitus. In: Walker BR, Colledge NR, Ralston SH et al. Davidson's Principles \&practice of Medicine, 22nd ed. Edinburgh: Elsevier; 2014:797-836.

6. Powers AC, Niswender KD, Evans-Molina C. Diabetes mellitus: diagnosis, classification and pathology. In: Jameson JL, Fauci AS, Kasper DL et al,

Harrison's Principles Of Internal Medicine, $20^{\text {th }}$ ed. New York: McGrow-Hill Education; 2018:2850-2859.

7. World Health Organization. Country and regional data on diabetes: WHO Eastern Mediterranean Region [Internet] .Geneva, Switzerland; 2020 [Cited 2020 Aug 25]. Available at: https://www.who.int/diabetes/facts/world_ figures/en/index2.html.

8. Al-Obaidi S, Abdulrazaq M, Muhammed S et al. Diagnosis of diabetes mellitus in patients with tuberculosis. In: Iraqi MOH National Tuberculosis Program. Tuberculosis in Patients with Diabetes Mellitus: A guide of management, $1^{\text {st }}$ ed. Iraq: $\mathrm{MOH} ; 2$ 2019:2-3: 7-8. Available at: http://phd.iq/Lion/mages/ PDFStore/Tuberculosis\%20in\%20Patients\%20with\%20DM\%20final.pdf

9. Powers AC, Stafford JM, Rickels MR. Diabetes mellitus: complications. In: Jameson JL, Fauci AS, Kasper DL et al, Harrison's Principles of Internal Medicine, $20^{\text {th }}$ ed. New York: McGrow-Hill Education; 2018:2875-2883.

10. Crandall J, Shamoon H. Diabetes Mellitus. In: Goldman L, Shafer Al, Goldman-Cesil Medicine, 25 th ed. Philadelphia: Elsevier; 2016:1527-1548.

11. Bargman JM, Skorecki KL. Chronic kidney disease. In: Jameson JL, Fauci AS, Kasper DL, et al, Harrison's Principles of Internal Medicine, 20 ${ }^{\text {th }}$ ed. New York: McGrow-Hill Education; 2018:2111-2121. 
12. Gale E, Anderson J. Diabetes mellitus. In: Kumar P, Clark M, Kumar \& Clark's Clinical Medicine, $9^{\text {th }}$ ed. Edinburgh: Elsevier; 2017:1241-1276.

13. Alicic R, Rooney M, Tuttle K. Diabetic kidney disease: challenges, progress, and possibilities. Clin J Am Soc Nephrol 2017;12:2032-2045. Available at: https://www.ncbi.nlm.nih.gov/pmc/articles/PMC5718284/.

14. Karalliedde J, Viberti G. Microalbuminuria and cardiovascular risk. AJH 2004;17:986-993. Available at: https://academic.oup.com/ajh/ article/17/10/986/107224.

15. Alaini A, Malhotra D, Rondon-Berrios $\mathrm{H}$, et al. Establishing the presence or absence of chronic kidney disease: uses and limitations of formulas estimating the glomerular filtration rate. World J Methodol 2017;7(3):73-92. Available at: http://dx.doi.org/10.5662/wjm.v7.i3.73.

16. Grill A, Brimble S. Approach to the detection and management of chronic kidney disease: what primary care providers need to know. Can Fam Physician 2018;64(10):728-735. Available at: https://www.ncbi.nlm.nih.gov/ pmc/articles/PMC6184972/.

17. Upadhyay $\mathrm{A}$, Inker L, levey A. Chronic kidney disease: definition, classification and approach to management. In: Turner N, Lameire N, Goldsmith D, et al. Oxford Textbook of Clinical Nephrology, $4^{\text {th }}$ ed. Glasgow: Bell \&Bain; 2016:743-755.

18. Levey A. Becker C, Inker, L. Glomerular filtration rate and albuminuria for detection and staging of acute and chronic kidney disease in adults: a systematic review. JAMA 2015;313(8):837-846. Available at: https://www. ncbi.nlm.nih.gov/pmc/articles/PMC4410363/.

19. Mitch W. Chronic kidney disease. In: Goldman L, Shafer A, Goldman-Cesil Medicine, 25 ${ }^{\text {th }}$ ed. Philadelphia: Elsevier; 2016:833-841.

20. Berl T, Henrich, W. Kidney-heart interactions: epidemiology, pathogenesis, and treatment. Clin J Am Society Nephrol 2006;1:8-18. Available at: https:// cjasn.asnjournals.org/content/1/1/8.

21. Goldsmith D. Cardiovascular disease and chronic kidney disease in the developed world. In: Turner N, Lameire N, Goldsmith D, et al. Oxford Textbook of Clinical Nephrology, $4^{\text {th }}$ ed. Glasgow: Bell \& Bain; 2016:777-780.

22. Yaqoob M, Ashman N. Kidney and urinary tract disease. In: Kumar P, Clark M, Kumar \& Clark's Clinical Medicine, $9^{\text {th }}$ ed. Edinburgh: Elsevier; 2017:723-794.

23. Levey A, Stevens $L$, Schmid $C$ et al. A new equation to estimate glomerular filtration rate. Ann Intern Med 2009;150(9):604-612. Available at: https:// www.ncbi.nlm.nih.gov/pmc/articles/PMC2763564/.

24. Ravera, M, Noberasco G, Weiss U, et al. CKD awareness and blood pressure control in the primary care hypertensive population. Am J Kidney Dis 2010;57(1):71-77. Available at: https://www.ajkd.org/article/S02726386(10)01395-1/fulltext.

25. McFarlane P, Cherney D, Gilbert R, et al. Chronic kidney disease in diabetes. CJD 2018;42:201-209. Available at: https://www.canadianjournalofdiabetes. com/article/S1499-2671(17)30985-1/fulltext.

26. Komenda P, Ferguson T, Macdonald K, et al. Cost-effectiveness of primary screening for CKD: a systematic review. Am J Kidney Dis 2014;63(5):789-797. Available at: https://www.ajkd.org/article/S0272-6386(14)00024-9/fulltext.

27. Du Bois D, Du Bois E. A formula to estimate approximate surface area if height and weight be known. Arch Int. Med 1917:17:863-871. Available at: https://www.scirp.org/(S(i43dyn45teexjx455qlt3d2q))/reference/ ReferencesPapers.aspx?ReferencelD=1332732.

28. Levey AS, Atkins R, Coresh J, et al. Chronic kidney disease as a global public health problem: approaches and initiatives - a position statement from kidney disease improving global outcomes. Kidney Int 2007;72(3):247-259. Available at: https://www.kidney-international.org/article/S00852538(15)52649-5/fulltext.

29. Stevens L, Fares G, Fleming J, et al. Low rates of testing and diagnostic codes usage in a commercial clinical laboratory: evidence for lack of physician awareness of chronic kidney disease. JASN 2005;16(8):2439-2448. Available at: https://jasn.asnjournals.org/content/16/8/2439.

30. Narenpitak S, Narenpitak A. Prevalence of chronic kidney disease in type 2 diabetes in primary health care unit of Udon Thani province, Thailand. J Med
Assoc Thai 2008;91(10):1505-1513. Available at: http://citeseerx.ist.psu.edu/ viewdoc/download?doi=10.1.1.463.7206\&rep=rep1\&type=pdf.

31. Zaman S. Detection of chronic kidney disease by using different equations of glomerular filtration rate in patients with type 2 diabetes mellitus: a cross-sectional analysis. Cureus 2017;9(6):e1352. Available at: https://www. ncbi.nlm.nih.gov/pmc/articles/PMC5510968/.

32. Bouzid C, Smida H, Kacem A, et al. Renal failure in Tunisian patients with type 2 diabetes: frequency and related factors. La Tunisie Med 2011;89(1):10-15. Available at: https://www.latunisiemedicale.com/articlemedicale-tunisie_1502_en.

33. Fiseha T, Kassim M, Yemane T. Chronic kidney disease and under diagnosis of renal insufficiency among diabetic patients attending a hospital in Southern Ethiopia. BMC Nephrol 2014;15:198. Available at: https:// bmcnephrol.biomedcentral.com/track/pdf/10.1186/1471-2369-15-198.

34. De Boer I, Rue T, Hall Y, et al. Temporal trends in the prevalence of diabetic kidney disease in the United States. JAMA 2011;305(24):2532-2539. Available at: https://www.ncbi.n/m.nih.gov/pmc/articles/PMC3731378/.

35. Nakata J, Ohsawa I, Onda K. et al. Risk of overestimation of kidney function using GFR-estimating equations in patients with low insulin clearance. J Clin Lab Anal 2012;26(4):248-253. Available at: https://www.ncbi.n/m.nih.gov/ pmc/articles/PMC6807624/.

36. Bradshaw C, Kondal D, Montez-Rath M, et al. Early detection of chronic kidney disease in low-income and middle-income countries: development and validation of a point-of-care screening strategy for India. BMJGH 2019;4(5):e001644. Available at: https://gh.bmj.com/content/4/5/e001644 long.

37. Hooi LS, Ong LM, Ahmad G., et al. A population-based study measuring the prevalence of chronic kidney disease among adults in West Malaysia. Kidney Int 2013;84(5):1034-1040. Available at: https://www.kidney-international. org/article/S0085-2538(15)56071-7/fulltext.

38. Jolly S, Navaneethan S, Schold J, et al. Chronic kidney disease in electronic health record problem list: quality of Care, ESRD, and mortality. Am J Nephrology 2014;39:288-296. Available at: https://www.karger.com/ Article/Fulltext/360306

39. Kazancioglu R. Risk factors for chronic kidney disease: an update. Kidney Int Suppl 2013;3(4):368-371. Available at: https://www.ncbi.nlm.nih.gov/pmc/ articles/PMC4089662/.

40. Hemmelgarn B, Zhang J, Manns B, et al. Progression of kidney dysfunction in community-dwelling elderly. Kidney Int. 2006;69(12):2155-2161. Available at: https://www.kidney-international.org/article/S00852538(15)51433-6/fulltext.

41. Ene-lordache B, Perico N, Bikbov B, et al. Chronic kidney disease and cardiovascular risk in six regions of the world (ISN-KDDC): a cross-sectional study. Lancet Glob Health 2016;4(5):307-319. Available at: https://www. thelancet.com/journals/langlo/article/PIIS2214-109X(16)00071-1/fulltext

42. Parameswaran S, Geda S, Rathi M, et al. Referral pattern of patients with end-stage renal disease at a public sector hospital and its impact on outcome. Natl Med J India 2011;24(4):208-213. Available at: https:// pubmed.ncbi.nlm.nih.gov/22208139/.

43. George C, Mogueo A, Okpechi l, et al. Chronic kidney disease in low-income to middle-income countries: the case for increased screening. BMJ Glob Health 2017;2:e000256. Available at: https://gh.bmj.com/content/2/2/ e000256.

44. Mansour A, Douri F. Diabetes in Iraq: facing the epidemic. A systematic review. Wulfenia 2015;22(3):258-273. Available at:https://www.researchgate. net/publication/280084146_Diabetes_in_Iraq_Facing_the_Epidemic_A_ systematic_Review.

45. Kitiyakara C, Yamwong S, Vathesatogkit $P$, et al. The impact of different GFR estimating equations on the prevalence of CKD and risk groups in a Southeast Asian cohort using the new KDIGO guidelines. BMC Nephrol 2012;13:1. [Cited 2020 Aug 25]. Available at: https://bmcnephrol. biomedcentral.com/articles/10.1186/1471-2369-13-1. 